\title{
Blackish Pigmentation of the Aorta in Patient with Alkaptonuria and Heyde's Syndrome
}

\author{
Fabio Capuano, MD, PhD*, Emiliano Angeloni, MD, Antonino Roscitano, MD, \\ Roberto Bianchini, MD, Simone Refice, MD, Andrea Lechiancole, MD, \\ Giovanni Melina, MD, PhD, Cosimo Comito, MD, Riccardo Sinatra, MD \\ Department of Cardiac Surgery, "Sapienza", Università di Roma, Sant'Andrea Hospital, Rome, Italy
}

\begin{abstract}
Alkaptonuria is an autosomal recessive trait resulting in an error of aromatic amino acids metabolism. Heyde's syndrome is a condition clustering together aortic valve stenosis and gastrointestinal bleeding from colonic angiodysplasia. At present, there is no report describing the association of the latter two syndromes in the same patient.

Here we present the case of a patient with severe aortic stenosis, alkaptonuria, and Heyde's syndrome. The patient underwent aortic valve replacement by means of a valvular bioprosthesis and the histological examination of the aortic cusps revealed calcific degeneration. This was associated with stromal degeneration characterized by extra-cellular deposition of granular, brownish-pigmented material along with macrophages and multiple foci of calfication showing the same brownish pigmentation. This configuration represents the typical pattern of homogentisic acid accumulation known as ochronosis.

The postoperative course was uneventful and the echocardiographic follow-up at 6 months postoperatively showed good-functioning of the aortic valve bioprosthesis.

Copyright $\odot 2014$ Science International Corp.
\end{abstract}

\section{Key Words}

Aortic valve replacement - Alkaptonuria - Heyde's syndrome

Alkaptonuria, an autosomal recessive trait, is a rare inborn error of metabolism of aromatic amino acids

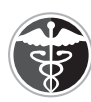

Fax +1 2037853346

E-Mail: aorta@scienceinternational.org

http://aorta.scienceinternational.org (c) 2014 Aorta.

Published by Science International Corp. ISSN 2325-4637

Accessible online at:

http://aorta.scienceinternational.org phenylalanine and tyrosine caused by deficiency of homogentisate 1,2-dioxygenase (HGO) activity in the liver; it is estimated to affect between 1 in 250,000 and 1 in 1,000,000 of the general population [1], and such a deficiency of HGO activity has been mapped to chromosome 3q21-q23, with more than 80 mutations discovered $[1,2]$.

Alkaptonuria enjoys the historic distinction of being one of the first conditions in which mendelian recessive inheritance was proposed, and of being one of the four conditions in the charter group of inborn errors of metabolism. The manifestations are urine that turns dark on standing and alkalinization, black ochronotic pigmentation of cartilage and collagenous tissues, and arthritis, especially characteristic in the spine [2]. Therefore, three major features of alkaptonuria are increased urine level of homogentisic acid (urine darkness after exposure to air is often seen as a result of oxidation processes), homogentistic acid accumulation with bluish-black pigmentation in connective tissues, and arthritis of the spine and the diarthrodial joints. Other effects of ochronosis include stones (renal, prostatic, gallbladder, and salivary), ruptures (muscle, tendons, and ligaments), osteopenia, and fractures [3].

Heyde's syndrome is a condition clustering together aortic valve stenosis and gastrointestinal bleeding from colonic angiodysplasia [4]. It was first

${ }^{*}$ Corresponding author:

Fabio Capuano, MD, PhD

Sapienza, Università di Roma

Sant'Andrea Hospital

Via di Grottarossa 1035

Rome 00189, Italy

Tel: +39 063377 5310, Fax: +39063 377 6317, E-mail: fabiocapuano75@gmail.com 


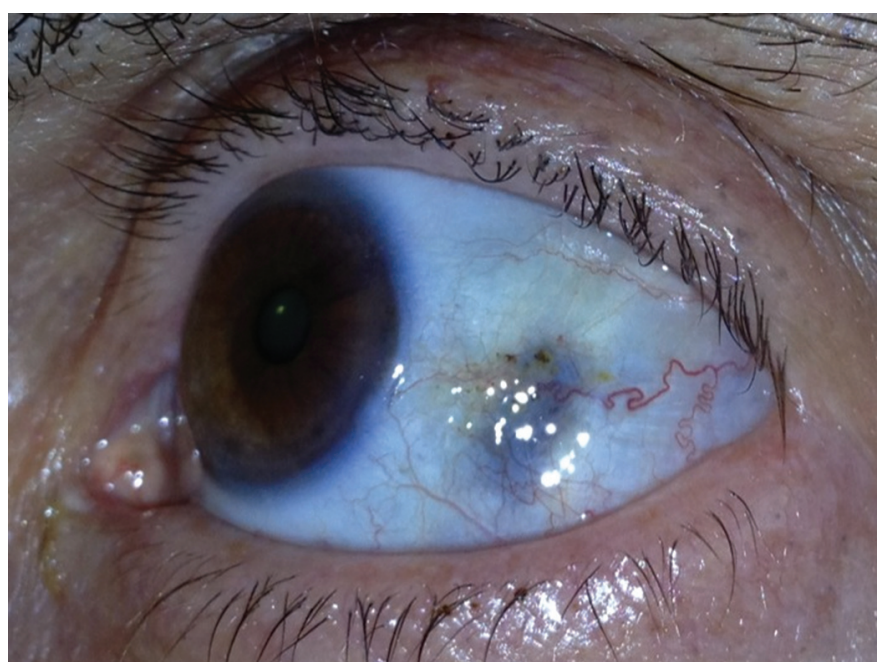

Figure 1. Scleral pigmentation due to alkaptonuria.

described by Heyde in 1958, and afterward it was discovered to be due to the induction of von Willebrand disease type IIA by valvular stenosis [5]. In these settings, gastrointestinal hemorrhage may present as hematemesis, melena, or hematochezia; platelet function analysis (with adenosine diphosphate closure time) or von Willebrand factor electrophoresis identifies reduction of ultralarge von Willebrand factor [5]. Elective treatment of Heyde's syndrome is aortic valve replacement [4-6].

To the best of our knowledge, there is no report describing the association of the latter two syndromes (alkaptonuria and Heyde's syndrome) in the same patient.

Here we present the case of a 67-year-old man who was admitted to our institution with the diagnosis of severe aortic valve stenosis, and with history of alkaptonuria and Heyde's syndrome.

Family history was negative with respect to both alkaptonuria and Heyde's syndrome. At admission, physical examination revealed blue-gray pigmented macules over the cartilaginous portions of the ears and on the sclera (Fig. 1). The patient relayed the history of spinal arthritis and recurrent hematemesis; furthermore, the chromatographic examination of urine revealed the presence of homogentisic acid, and a gastroscopy revealed angiodysplasia in the upper gastrointestinal tract. Electrophoresis of the von Willebrand factor was not available at the time of intervention. Preoperative renal function was normal (creatinine clearance $75 \mathrm{~mL} / \mathrm{min}$ ) and abdominal echo to investigate kidney morphology was not performed.
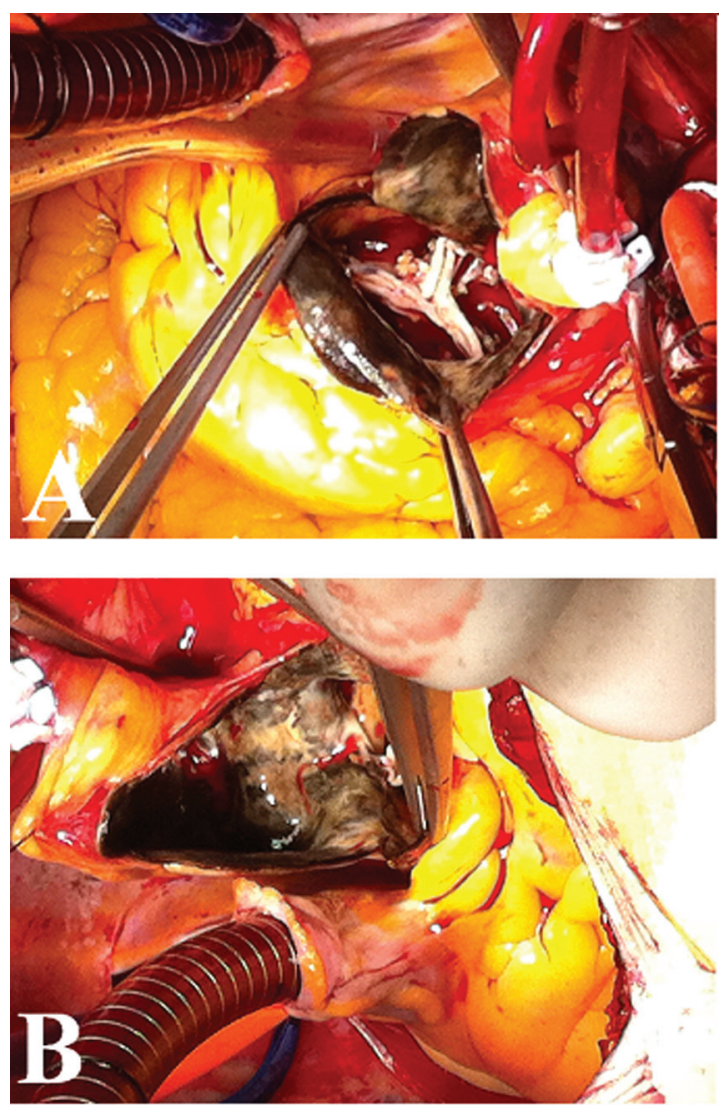

Figure 2. Blackish aortic wall (A, B). Aortic leaflet calcification (A).

The aortic valve stenosis had become symptomatic with syncopal episodes in the past 2 months. The patient was not taking any medicine. Cardiac cardiac catheterization demonstrated severe calcification of the aortic leaflets with an aortic valve area of 0.4 $\mathrm{cm}^{2}$. Preoperative echocardiography showed severe aortic stenosis with mean transvalvular gradient of $69 \mathrm{~mm} \mathrm{Hg}$ and aortic valve area of $0.51 \mathrm{~cm}^{2}, \mathrm{a}$ maximum diameter of the ascending aorta of $41 \mathrm{~mm}$, and a maximum diameter of the bulb of $34 \mathrm{~mm}$.

At surgery it was noted that the inside wall of the aorta was entirely covered with black patches (Fig. 2), and the aortic valve leaflets were severely calcified. The native aortic valve was replaced with a biological prosthesis $(21 \mathrm{~mm}$ Edwards Magna Aortic valve prosthesis, Edwards Lifesciences, Irvine, CA). The optimal choice of the type of heart valve to be used is yet to be explored in patients with alkaptonuria. We chose a bioprosthetic valve, considering the age of the patient, and the decision was guided by the patient's 


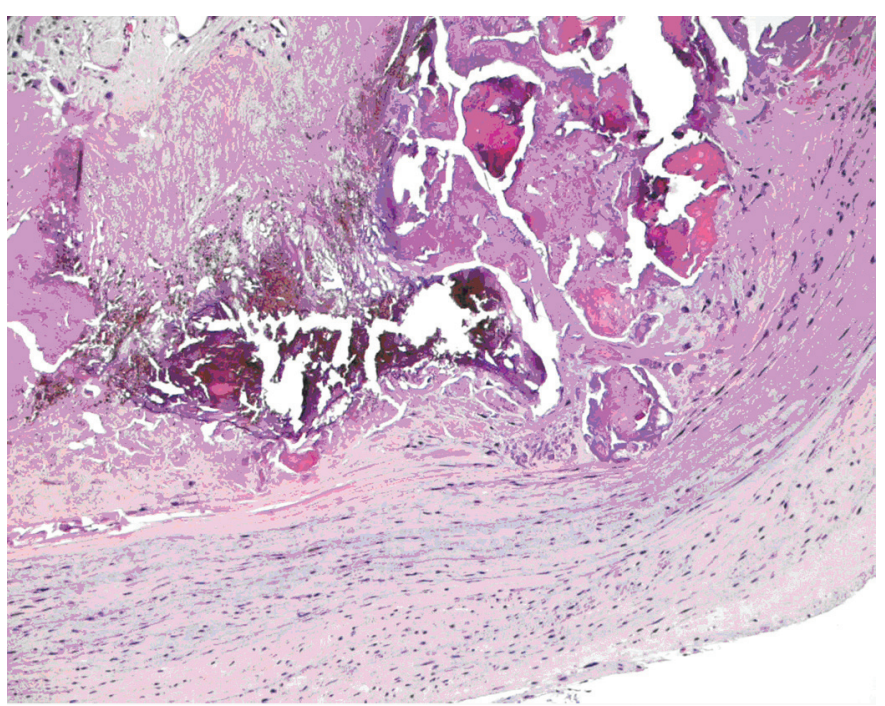

Figure 3. Microscopic pattern of the native aortic cusp. Calcific degeneration was associated with stromal degeneration characterized by extra-cellular deposition of granular, brownish-pigmented material along with macrophages and multiple calfication foci showing the same brownish pigmentation.

choice after careful explanation of the risks/benefits ratio.

Histological examination of the aortic cusps revealed calcific degeneration, associated with stromal degeneration characterized by extracellular deposition of granular, brownish-pigmented material along with macrophages and multiple calcification foci showing the same brownish pigmentation (Fig. 3). Histologic report on the aortic wall also showed extracellular deposition of granular, brownish-pigmented material, mostly at the subintimal level. This pattern was typical of homogentisic acid accumulation (ochronosis), as described in alkaptonuric patients in previous reports $[1,3]$.

The postoperative course was uneventful, and gastrointestinal bleeding disappeared. The patient entered a close echocardiographic follow-up program to control for prosthetic valve calcification. We did not consider using nitisinone (an inhibitor of the tyrosine pathway, found associated with a significant reduction of plasma homogentisic acid levels, and probably useful in preventing calcification progression) [7] because of the lack of reports regarding alkaptonuric aortic valve disease in the replaced bioprosthetic valve of patients with concomitant Heyde's syndrome. The last echocardiographic follow-up (6 months postoperatively) showed good functioning of the aortic valve bioprosthesis (mean transprosthetic gradient 12 $\mathrm{mm} \mathrm{Hg}$ ).

\section{Conflict of Interest}

The authors have no conflict of interest relevant to this publication.

Comment on this Article or Ask a Question

\section{References}

1. Roser M, Moller J, Komoda T, Knosalla C, Stawowy P. Alkaptonuric aortic stenosis. Eur Heart J. 2008;29:444. 10.1093/eurheartj/ehm406

2. Online Mendelian Inheritance in Man, MIM\# 203500; http://omim.org/entry/203500

3. Pettit SJ, Fisher M, Gallagher JA, Ranganath LR. Cardiovascular manifestations of alkaptonuria. J Inherit Metab Dis. 2011;34:11771181. 10.1007/s10545-011-9339-z

4. Saad RA, Lwaleed BA, Kazmi RS. Gastrointestinal bleeding and aortic stenosis (Heyde syndrome): the role of aortic valve replacement. J Card Surg. 2013;28:414-416. 10.1111/jocs.12131
5. Vincentelli A, Susen S, Le Tourneau T, Six I, Fabre $\mathrm{O}$, Juthier $\mathrm{F}$, et al. Acquired von Willebrand syndrome in aortic stenosis. N Engl J Med. 2003; 349:343-349. 10.1056/NEJMoa022831

6. Abi-akar R, El-rassi I, Karam N, Jassar Y, Slim R, Jebara V. Treatment of Heyde's syndrome by aortic valve replacement. Curr Cardiol Rev. 2011;7:47-49. 10.2174/157340311795677699

7. Introne WJ, Perry MB, Troendle J, Tsilou E, Kayser MA, Suwannarat $P$, et al. A 3-year randomized therapeutic trial of nitisinone in alkaptonuria. Mol Genet Metab. 2011;103: 307-314. 10.1016/j.ymgme.2011.04.016
Cite this article as: Capuano $F$, Angeloni E, Roscitano A, Bianchini R, Refice $S$, Lechiancole A, Melina G, Comito C, Sinatra R. Blackish Pigmentation of the Aorta in Patient with Alkaptonuria and Heyde's Syndrome. Aorta 2014;2(2): 74-76. DOl: http://dx.doi.org/10.12945/ j.aorta.2014.13-058 